\title{
Air embolism during off-pump coronary artery bypass graft surgery -A case report-
}

\section{Choo-Hoon Chang, Young Hee Shin, and Hyun-Sung Cho}

Department of Anesthesiology and Pain Medicine, Samsung Medical Center, Sungkyunkwan University School of Medicine, Seoul, Korea

There have been several reports of gas embolism occurring during off-pump coronary artery bypass graft (OPCAB) surgery. However, all these cases of air embolism were associated with the repair of venous circulation, using a $\mathrm{CO}_{2}$ blower. In this report, we describe a rare case of air embolism in the coronary arteries associated with the use of a $\mathrm{CO}_{2}$ blower during OPCAB. There was no injury to the veins during OPCAB. The air embolism was treated successfully with cardiopulmonary bypass. (Korean J Anesthesiol 2012; 63: 72-75)

Key Words: Air embolism, Catheterization, Off-pump coronary artery bypass, Swan-Ganz.

Air embolism is a rare complication that may result from procedures performed by clinicians in a variety of settings. There have been several reports of gas embolism occurring during off-pump coronary artery bypass graft (OPCAB) surgery. However, all these cases of air embolism were associated with the repair of venous circulation. During endoscopic saphenectomy or anastomosis for an injured coronary vein, an air embolism was caused by the use of a $\mathrm{CO}_{2}$ blower.

In this report, we describe a rare case of air embolism in the coronary arteries associated with the use of a $\mathrm{CO}_{2}$ blower at the end of the OPCAB.

\section{Case Report}

A 73-year-old man, $159 \mathrm{~cm}$ in height and $59 \mathrm{~kg}$ in weight with unstable angina pectoris was scheduled for OPCAB surgery. He had hypertension, so he had been taking aspirin, clopidogrel, and olmesartan since the last 4 years.

His preoperative coronary angiogram showed $75 \%$ stenosis of the proximal to mid left anterior descending artery (LAD), $40 \%$ stenosis of the distal left circumflex artery (LCx), and total occlusion of the proximal right circumflex artery (RCA). A stent had been inserted in the proximal RCA 3 months ago. After the procedure, the left ventricular function was preserved, with an ejection fraction of $63 \%$ on echocardiography. And a first-degree atrioventricular (AV) block was observed in the electrocardiogram (ECG). There was no remarkable finding in the other preoperative laboratory studies and chest X-ray.

On arrival, continuous monitoring of ECG lead II and $\mathrm{V}_{5}$ was initiated, and the left radial artery cannulation was done

Received: May 19, 2011. Revised: July 11, 2011. Accepted: July 20, 2011.

Corresponding author: Young Hee Shin, M.D., Department of Anesthesiology and Pain Medicine, Samsung Medical Center, Sungkyunkwan University School of Medicine, Irwon-dong, Gangnam-gu, Seoul 135-710, Korea. Tel: 82-2-3410-0784, Fax: 82-2-3410-6626, E-mail: tuderi02@ naver.com

(C) This is an open-access article distributed under the terms of the Creative Commons Attribution Non-Commercial License (http:// creativecommons.org/licenses/by-nc/3.0/), which permits unrestricted non-commercial use, distribution, and reproduction in any medium, provided the original work is properly cited. 
under local anesthesia for pressure monitoring. Anesthesia was induced with intravenous injection of midazolam $3 \mathrm{mg}$, etomidate $20 \mathrm{mg}$, sufentanil $100 \mathrm{ug}$, and rocuronium $50 \mathrm{mg}$. Anesthesia was maintained with $2 \mathrm{~L} / \mathrm{min}$ of oxygen and 1.2-2.5 vol\% isoflurane. After the induction of anesthesia, a Swan-Ganz (S-G) introducer was inserted in the right internal jugular vein, and a pulmonary artery catheter (PAC) was placed through the S-G introducer. Initial hemodynamic parameter values were as follows: pulmonary artery pressure (PAP) 26-17-13 $\mathrm{mmHg}$, central venous pressure (CVP) $6 \mathrm{mmHg}$, cardiac output (CO) $3.8 \mathrm{~L} / \mathrm{min}$, and cardiac index (CI) $2.3 \mathrm{~L} / \mathrm{min} / \mathrm{m}^{2}$. Lastly, a transesophageal echocardiographic (TEE) probe was inserted.

Median sternotomy was performed followed by the dissection and preparation of both the internal thoracic arteries. The patient was placed in the Trendelenburg position and the surgeon anastomosed the vessels using a $\mathrm{CO}_{2}$ blower to maintain a clear surgical field. During the operation, his vital signs were stable with heart rate (HR) at $55-80$ beats $/ \mathrm{min}$, blood pressure (BP) between 70-120/35-70 $\mathrm{mmHg}$, oxygen saturation $\left(\mathrm{SpO}_{2}\right)$ between $98-100 \%$, and end tidal $\mathrm{CO}_{2}\left(\mathrm{ETCO}_{2}\right)$ between 28-32 mmHg. Arterial blood gas analysis (ABGA) was done after anastomosis of coronary arteries, and the results showed, a pH of 7.367, $\mathrm{PaO}_{2}$ of $347.5 \mathrm{mmHg}$, and $\mathrm{PaCO}_{2}$ of 48.4 mmHg with an $\mathrm{FiO}_{2}$ of 1.0.

After the end of the main procedure, the sternum was wired together in the Trendelenburg position, and the TEE probe was removed. Just after the sternum was closed, the patient was returned to the supine position. And then, the monitoring parameters changed abruptly. BP decreased from 105/55 $\mathrm{mmHg}$ to $55 / 30 \mathrm{mmHg}, \mathrm{ETCO}_{2}$ decreased from 32 to $15 \mathrm{mmHg}$, PAP increased from 30-21-15 $\mathrm{mmHg}$ to 56-38-27 $\mathrm{mmHg}$, and $\mathrm{SpO}_{2}$ dropped from $100 \%$ to $53 \%$. Soon afterwards, the

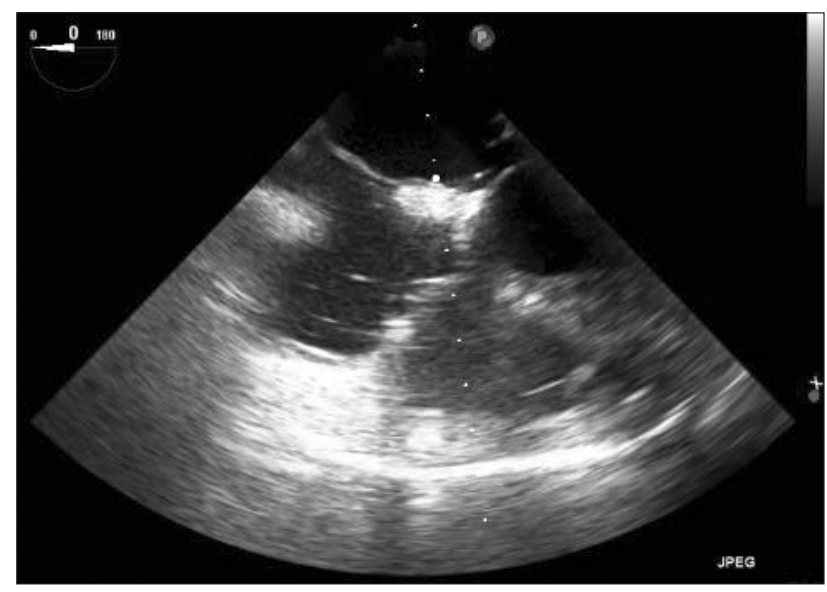

Fig. 1. Transesophageal echocardiographic 2-dimensional examination from the midesophageal 4-chamber view. There are gas bubbles in the right atrium and ventricle. patient became asystolic as was noted on the ECG. The patient was changed from the supine position to the Trendelenburg position, and was ventilated with $100 \%$ oxygen on suspicion of air embolism. The surgeons reopened the sternum and started open cardiac massage immediately. Epinephrine $1 \mathrm{mg}$ and atropine $0.5 \mathrm{mg}$ were administered initially and epinephrine 1 mg was administered repeatedly after every 3 minutes. Normal sinus rhythm returned four minutes after the initiation of cardiopulmonary resuscitation (CPR). However immediately afterwards, ventricular fibrillation developed,, and so we gave a DC cardioversion shock with $20 \mathrm{~J}$. Soon, normal sinus rhythm returned. The TEE probe was reinserted and air bubbles were observed in all chambers of the heart (Fig. 1). Although BP increased to $150 / 80 \mathrm{mmHg}$ and oxygen saturation increased to $90 \%$, PAP did not decrease. ABGA showed a $\mathrm{pH}$ of 7.409, $\mathrm{PaO}_{2}$ of $387.1 \mathrm{mmHg}$, and $\mathrm{PaCO}_{2}$ of $41.6 \mathrm{mmHg}$ with an $\mathrm{FiO}_{2}$ of 1.0.

The operators started preparation for a cardiopulmonary bypass (CPB), and the Trendelenburg position was maintained. Within 10 minutes after cardiac arrest, aorta, and superior vena cava were cannulated. Although hemodynamic stability was achieved, partial CPB was started for removing the air. Then we removed the PAC from the S-G introducer because of the suspicion of air entering through it. On examination, no defect or abnormality was found in the catheter and the connections. Finally, the patient was weaned uneventfully from CPB and the operation was completed.

The postoperative course was unremarkable. No neurological abnormalities and ischemic changes were noted in the patient. He was discharged on the $17^{\text {th }}$ postoperative day.

\section{Discussion}

This was the first case which reported that the use of a $\mathrm{CO}_{2}$ blower during the OPCAB procedure concomitant with the change in the patient's position caused massive air emboli. Air embolism occurred just at the same time when the sternum was wired together and patient's position was changed from the Trendelenburg position to the supine position. Besides, there were no specific signs of air entering through the catheter, and the CVP was normal at that time. Increased thoracic pressure due to sternal wiring and the change in the patient's position might have caused air diffusion from some parts of the heart. But, we could not see the air on TEE because the TEE probe had already been removed by that time. This is a limitation of this case report.

In this case, two factors were considered to cause massive air emboli in the heart during OPCAB. One was the problem in the catheter, and the other was the $\mathrm{CO}_{2}$ blower used.

First, we excluded the problem in the catheter. A problem in the S-G introducer, PAC, or the connection between the two 
devices could cause air to enter into the internal jugular vein (IJV) directly $[1,2]$. Air embolism caused by the S-G introducer was preferentially considered because there have been a few reported cases of air embolism with the S-G introducer kit [1]. However, air embolism was not observed either on transesophageal echocardiography or clinical findings although we had not removed the S-G introducer after the embolic event. Therefore, we could exclude S-G introducer as the cause of air embolism. In addition, although a problem in the one-way valve between the S-G introducer and PAC could also cause an air embolism [2], this cause could be excluded because the front part of this connection was locked and sealed with tape. Furthermore, a problem related with the PAC was also excluded because no gross abnormality was observed after the removal of PAC. The CVP and PAP were normal before the cardiac arrest, so these pressures could rather cause bleeding than air entry. But there was neither any evidence of backward blood flow nor any space for air entry.

Second, the $\mathrm{CO}_{2}$ blower was considered as another cause of the pulmonary air embolism. There have been a few reports demonstrating that a $\mathrm{CO}_{2}$ gas blower could cause coronary artery ischemia and pulmonary air embolism [3-5]. In a case of coronary artery ischemia, the spray pressure can exceed the diastolic pressure, especially during grafting at the lateral or posterior wall when hemodynamic compromise due to mechanical heart displacement is most severe [3]. In this situation, $\mathrm{CO}_{2}$ blowing at an incompletely slinged coronary arteriotomy site can result in retrograde migration of $\mathrm{CO}_{2}$ into the ascending aorta, causing coronary embolism in the right coronary artery. And in a case of pulmonary air embolism, the main cause of air embolism may be the $\mathrm{CO}_{2}$ blower used to repair a torn coronary vein instead of an artery or torn right ventricle outflow track (RVOT) $[4,5]$. Because the venous pressure is much lower than the arterial pressure, $\mathrm{CO}_{2}$ directly enters the venous circulation easily. Even though in this case, the operator did not use a $\mathrm{CO}_{2}$ blower to repair any torn coronary vein or RVOT, a $\mathrm{CO}_{2}$ blower is one of the possible causes of air embolism.

During OPCAB, bleeding from a coronary artery may interrupt the construction of a distal anastomosis. To facilitate visualization of the anastomotic site, several techniques have been used including a coronary shunt or occluders, intermittent irrigation with normal saline, or a gas blower. In particular, a gas blower using $\mathrm{CO}_{2}$ is more advantageous with regard to solubility in blood, and has been safely used to provide a dry field during distal coronary anastomosis [6]. However, blowing of compressed $\mathrm{CO}_{2}$ during $\mathrm{OPCAB}$ causes embolic complications. However, in the previous case, air entered through the venous system. The present case is a very rare case of air embolism caused without repair of torn venous system.
However, we are not sure how the $\mathrm{CO}_{2}$ gas used in the anastomosis of the coronary artery entered the RV cavity. We suspect two possible ways for this occurrence. First of all, the patient might have had a coronary artery fistula. Coronary artery fistulae are abnormal communications between a coronary artery and a cardiac chamber or a major vessel (vena cava, pulmonary veins, pulmonary artery) [7]. Small coronary artery fistulae that are not clinically detectable are not clearly associated with significant long-term complications. Medium or large fistulae are associated with significant long-term problems including angina, arrhythmias, myocardial infarction, endocarditis, and progressive dilation [8]. Coronary arteryvenous fistulae were considered as the cause of air embolism in this case. However, preoperative coronary angiography did not show any fistula. The other consideration was that the patient might have had a minimal patent foramen ovale (PFO). Of course, no PFO was observed in the preoperative and intraoperative echocardiography and that is why we excluded the possibility that the air could have entered from the venous side. But when the patient's position was changed abruptly, the BP and CVP might have dropped at the moment. Then, a massive influx of air from the venous system might have passed through the minimal PFO, which could have been the cause of a situation like in this case.

This case followed the typical pattern of $\mathrm{CO}_{2}$ embolism. Dissolved $\mathrm{CO}_{2}$ increases $\mathrm{EtCO}_{2}$, and then undissolved $\mathrm{CO}_{2}$ forms bubbles that increase physiologic dead space, leading to pulmonary hypertension, oxygen desaturation, with reduced $\mathrm{EtCO}_{2}$. Sustained pulmonary air embolism may lead to cardiac failure, systemic hypotension, and cardiovascular collapse through a gas-lock effect. Usually, microembolism of $\mathrm{CO}_{2}$ is well tolerated because of the rapid and complete solubility of $\mathrm{CO}_{2}$, but gross embolization may overwhelm the vasculature and cause devastating results [9].

Since the detection of air embolism is a clinical diagnosis, we should consider the diagnosis of air embolism in circumstances such as unexplained hypotension or decrease in $\mathrm{ETCO}_{2}$ intraoperatively in cases in which the operation is performed in the reverse Trendelenburg position or in situations where there is exposure of venous vasculature to atmospheric pressure [10]. A previous study on $\mathrm{EtCO}_{2}$ monitoring to detect $\mathrm{CO}_{2}$ embolism using a pig model showed that TEE is the "gold standard" monitor with earlier detection of gas emboli than $\mathrm{EtCO}_{2}$ change. Because $\mathrm{CO}_{2}$ is highly soluble in blood and rapidly absorbed from the blood-stream, there is also a time lag between TEE image and $\mathrm{EtCO}_{2}$ change during acute $\mathrm{CO}_{2}$ embolism [11]. Therefore, TEE is a more sensitive method to detect $\mathrm{CO}_{2}$ embolism than $\mathrm{EtCO}_{2}$ and mean PA pressure changes.

In our case, the PAC was removed because of the suspicion of air entering through it. Some authors have reported that 
aspiration of air from the right atrium via PAC was effective although PAC was less effective than Bunegin-Albin multiorifice catheter. As the average amount of air aspirated through the PAC was $15 \mathrm{ml}$, only the aspiration of air through the PAC could stabilize the patient hemodynamically in some cases [12]. However, in this case, patient became asystolic just after the suspicion of an air embolism was raised, so chest compression was applied and preparation of CPB was started immediately. Indeed, it took only 9 minutes from the occurrence of the hemodynamic changes to the initiation of CPB. Although CPB is the most effective method of removing the air from the heart, it should be considered as the last option. Initial treatments for air embolism are as follows; increase the inspiratory oxygen, hemodynamic support with dobutamine, norepinephrine, and isoproterenol, and central venous catheter aspiration. When the results obtained with the initial treatment are insufficient, chest compression and CPB should be considered. Considering this, one of the limitations of the management was that we did not aspirate air through the PAC before its removal.

In conclusion, anesthesiologists should all the time be careful of occurrence of an air embolism during OPCAB. TEE monitoring should be continued until the operation is completed. And if there are torn veins and torn in the right ventricle, another method to visualize the vessels including a coronary shunt or occluders should be considered. During anastomosis of arteries, we should pay careful attention to deairing and flushing of the graft to prevent air embolism.

\section{References}

1. Hartung EJ, Ender J, Sgouropoulou S, Bierl R, Engelhardt W, Engemann R. Severe air embolism caused by a pulmonary artery introducer sheath. Anesthesiology 1994; 80: 1402-3.

2. Doblar DD, Hinkle JC, Fay ML, Condon BF. Air embolism associated with pulmonary artery catheter introducer kit. Anesthesiology 1982; 56: 389-91.

3. Shim JK, Choi YS, Yoo KJ, Kwak YL. Carbon dioxide embolism induced right coronary artery ischaemia during off-pump obtuse marginalis artery grafting. Eur J Cardiothorac Surg 2009; 36: 598-9.

4. Hirata N, Kanaya N, Yamazaki Y, Sonoda H, Namiki A. Pulmonary embolism caused by a carbon dioxide blower during off-pump coronary artery bypass grafting. J Anesth 2010; 24: 114-6.

5. Lee JH, Yoon SZ, Choi JY, Bahk JH, Jeon Y. Massive carbon dioxide embolism caused by a carbon dioxide blower during the repair of a coronary vein during off-pump coronary artery bypass. J Cardiothorac Vasc Anesth 2007; 21: 715-7.

6. Burfeind WR Jr, Duhaylongsod FG, Annex BH, Samuelson D. Highflow gas insufflation to facilitate MIDCABG: effects on coronary endothelium. Ann Thorac Surg 1998; 66: 1246-9.

7. Luo L, Kebede S, Wu S, Stouffer GA. Coronary artery fistulae. Am J Med Sci 2006; 332: 79-84.

8. Latson LA. Coronary artery fistulas: how to manage them. Catheter Cardiovasc Interv 2007; 70: 110-6.

9. Martineau A, Arcand G, Couture P, Babin D, Perreault LP, Denault A. Transesophageal echocardiographic diagnosis of carbon dioxide embolism during minimally invasive saphenous vein harvesting and treatment with inhaled epoprostenol. Anesth Analg 2003; 96: 962-4.

10. Mirski MA, Lele AV, Fitzsimmons L, Toung TJ. Diagnosis and treatment of vascular air embolism. Anesthesiology 2007; 106: 164-77.

11. Couture P, Boudreault D, Derouin M, Allard M, Lepage Y, Girard D, et al. Venous carbon dioxide embolism in pigs: an evaluation of end-tidal carbon dioxide, transesophageal echocardiography, pulmonary artery pressure, and precordial auscultation as monitoring modalities. Anesth Analg 1994; 79: 867-73.

12. Mirski MA, Lele AV, Fitzsimmons L, Toung TJ. Diagnosis and treatment of vascular air embolism. Anesthesiology 2007; 106: 16477. 